\title{
A Role for the Calmodulin Kinase II-Related Anchoring Protein ( $\alpha$ kap) in Maintaining the Stability of Nicotinic Acetylcholine Receptors
}

\author{
Chakib Mouslim*, Mohamed Aittaleb*, Richard I. Hume, and Mohammed Akaaboune \\ Department of Molecular, Cellular, and Developmental Biology, University of Michigan, Ann Arbor, Michigan 48109
}

$\alpha$ kap, a muscle specific anchoring protein encoded within the Camk2a gene, is thought to play a role in targeting multiple calcium/ calmodulin kinase II isoforms to specific subcellular locations. Here we demonstrate a novel function of $\alpha$ kap in stabilizing nicotinic acetylcholine receptors (AChRs). Knockdown of $\alpha$ kap expression with shRNA significantly enhanced the degradation of AChR $\alpha$-subunits $(\mathrm{AChR} \alpha)$, leading to fewer and smaller AChR clusters on the surface of differentiated $\mathrm{C} 2 \mathrm{C} 12$ myotubes. Mutagenesis and biochemical studies in HEK293T cells revealed that $\alpha$ kap promoted $\mathrm{AChR} \alpha$ stability by a ubiquitin-dependent mechanism. In the absence of $\alpha \mathrm{kap}, \mathrm{AChR} \alpha$ was heavily ubiquitinated, and the number of $\mathrm{AChR} \alpha$ was increased by proteasome inhibitors. However, in the presence of $\alpha$ kap, $\mathrm{AChR} \alpha$ was less ubiquitinated and proteasome inhibitors had almost no effect on AChR $\alpha$ accumulation. The major sites of $\mathrm{AChR} \alpha$ ubiquitination reside within the large intracellular loop and mutations of critical lysine residues in this loop to arginine increased AChR $\alpha$ stability in the absence of $\alpha$ kap. These results provide an unexpected mechanism by which $\alpha$ kap controls receptor trafficking onto the surface of muscle cells and thus the maintenance of postsynaptic receptor density and synaptic function.

\section{Introduction}

The clustering of nicotinic acetylcholine receptors (AChRs) at the postsynaptic membrane is a hallmark of the neuromuscular junction (NMJ) (Sanes and Lichtman, 2001). The initial steps involved in the formation of receptor clusters have been studied extensively, and several key components of this multistep process have been identified. Based on mouse genetic studies, MuSK (muscle-specific tyrosine kinase), LRP4 (low-density lipoprotein receptor-related protein 4), rapsyn, and Dok7 (dedicator of cytokinesis family member 7) all have been shown to be required for the initial formation of receptor clusters (Gautam et al., 1995; DeChiara et al., 1996; Sanes and Lichtman, 2001; Okada et al., 2006; Weatherbee et al., 2006; Kim et al., 2008; Zhang et al., 2008; $\mathrm{Wu}$ et al., 2010). Components of the dystrophin glycoprotein complex have also been shown to be involved in clustering and in the maintenance of postsynaptic receptor density. For example, mice deficient in $\alpha$-dystrobrevin or $\alpha$-syntrophin exhibit dramatic reductions in AChR number and density and an abnormal pattern of receptor clustering (Adams et al., 2000; Grady et al., 2000; Martinez-Pena y Valenzuela et al., 2011).

\footnotetext{
Received Dec. 27, 2011; revised Feb. 19, 2012; accepted Feb. 20, 2012.

Author contributions: C.M., R.I.H., and M.Ak. designed research; C.M. performed research; M.Ai. contributed unpublished reagents/analytic tools; C.M., R.I.H., and M.Ak. analyzed data; C.M., R.I.H., and M.Ak. wrote the paper.

This work was supported by National Institutes of Health Grant NS-047332. The anti-AChR (mab35) antibodies were obtained from the Developmental Studies Hybridoma Bank. We thank Dr. Stephan Kröger and his group for providing antibodies and Lrp4 construct and members of our laboratory for their comments on this manuscript.

*C.M. and M.Ai. contributed equally to this work.

The authors declares no competing financial interests.

Correspondence should be addressed to Mohammed Akaaboune, Department of Molecular, Cellular, and Developmental Biology, 830 North University Avenue, Ann Arbor, Ml 48109. E-mail: makaabou@umich.edu.

DOI:10.1523/JNEUROSCI.6477-11.2012

Copyright $\odot 2012$ the authors $\quad 0270-6474 / 12 / 325177-09 \$ 15.00 / 0$
}

The maintenance of a high density of AChR in the postsynaptic membrane may also depend on the mechanisms that regulate their progression through the secretory pathway from the endoplasmic reticulum (ER) to the cell surface. However, the molecular machinery that controls the processing of AChR within the secretory pathway remains poorly defined. In an effort to understand the machinery involved in AChR trafficking, we used a multidisciplinary approach that led us to identify a novel function for $\alpha$ kap, a protein implicated previously in localizing multiple isoforms of calcium/calmodulin-dependent protein kinase II (CaMKII). $\alpha$ kap is a muscle-specific protein encoded within the Camk2a gene that contains a putative transmembrane domain and an association domain but lacks the catalytic domain that is responsible for the kinase activity of CaMKII subunit $\alpha$ (Bayer et al., 1996, 1998, 2002). In skeletal and cardiac muscles, it is thought that $\alpha$ kap is involved in targeting CaMKII isoforms to the sarcoplasmic reticulum in which they regulate calcium homeostasis (Campbell and MacLennan, 1982; Chu et al., 1990; Wang and Best, 1992; Xu et al., 1993; Hain et al., 1995; Pawson and Scott, 1997; Bayer et al., 1998; Nori et al., 2003). In mammalian muscle cells, at least three isoforms of CaMKII $\left(\beta_{\mathrm{m}}, \gamma\right.$, and $\left.\delta\right)$ have been found to be expressed (Damiani et al., 1995; Bayer et al., 1998) and concentrated at the NMJs (Martinez-Pena y Valenzuela et al., 2010). In the present study, we investigated the role of $\alpha$ kap in muscle cells by knocking down its expression with shorthairpin RNA (shRNA) and discovered that $\alpha$ kap has a novel function, regulating the degradation of AChR protein.

\section{Materials and Methods}

Plasmid constructs. Plasmids containing mouse AChR subunit cDNAs driven by the CMV promoter were a generous gift from Dr. William 
Green (University of Chicago, Chicago, IL), and Lrp4 cDNA was a generous gift from Dr. Stephan Kröger (University of Munich, Munich, Germany). Throughout the paper, the respective subunits are referred to as $\mathrm{AChR} \alpha, \mathrm{AChR} \beta, \mathrm{AChR} \delta$, and $\mathrm{AChR} \varepsilon$. The HA- $\alpha$ kap construct was a generous gift from Dr. Ulrich Bayer (University of Colorado, Denver, CO). GFP- $\alpha$ kap was generated using the primers 5' -GGAATTCTATGC TG CTCTTTCTCACGCTGTGG and 5' -GGGTACCATGCGGCAGGA CGGAGGGCGCCCCAGATCTGT and cloned into the pEGFP-C3 vector, in which alanine was mutated to lysine at position 207 (A207K) to prevent the dimerization of GFP (monomeric-GFP-C3).

A plasmid encoding mouse protein disulfide isomerase associated protein 3 (pdiA3) cDNA was purchased from Origen (MC200134) and subcloned into the mCherry-C1 vector (Clontech) using the following primers: 5'-GGGAATTCTATGCGCTTCAGCTGCCTAGC and 5'-GG GGTACCTTAGAGGTCCTCTTGTGCC.

Mutations of lysine residues (K359, K347, K388, and K393) to arginine within the intracellular loop of $\mathrm{AChR} \alpha$ were generated using the QuickChange XL Site-Directed Mutagenesis Kit (Stratagene). The following primers were used for mutagenesis: 359 forward, $5^{\prime}$-CTATGTCTTCTGTAAAAA TCCTTCTCTCTTGTTTATCTCTGGATGGTC; 359 reverse, $5^{\prime}$-GACCAT CCAGAGATAAACAAGAGAGAAGGATTTTTACAGAAGACATAG; 374 forward, $5^{\prime}$-GATATATCTGACATCTCTGGGAGACCGGGTCCTCCACC TATGGGC; 374 reverse, $5^{\prime}$-GCCCATAGGTGGAGGACCCGGTCTCCCA GAGATGTCAGATATATC; 388 forward, 5' -GGCTTTCACTCTCCGCTG ATCAGACACCCTGAGGTGAAAAGCGCC; 388 reverse, 5' -GGCGCTTT TCACCTCAGGGTGTCTGATCAGCGGAGAGTGAAAGCC; 393 forward, 5' -CACCCTGAGGTGAGGAGCGCCATCGAGGGG; and 393 reverse, $5^{\prime}$-CCCCTCGATGGCGCTCCTCACCTCAGGGTG.

Two shRNA constructs against murine $\alpha$ kap were generated by cloning the annealed target sequences (nucleotide starting position at either 137 or 337) into BglII/HindIII sites of the pSUPER vector or pSUPER. Neo.GFP according to the recommendations of the manufacturer. The target sequence of the shRNA to $\alpha$ kap at position 137 is $5^{\prime}$-GTTCAGTT AATGGAATCTTC and at position 337 is $5^{\prime}$-GGCCTGGACTTTCATC GATTC. A scrambled shRNA (as negative control) was also generated: 5'-GGTTATCTATTAGCGATTCTA. All shRNA constructs were verified by DNA sequencing.

Cell culture and transfection. The $\mathrm{C} 2 \mathrm{C} 12$ muscle cell line and the HEK293T human embryonic kidney cell line were purchased from the American Type Culture Collection. HEK293T cells were grown at $37^{\circ} \mathrm{C}$ in $5 \% \mathrm{CO}_{2}$ and maintained in DMEM containing $10 \%(\mathrm{v} / \mathrm{v})$ fetal bovine serum (FBS) (Invitrogen) and 1\% penicillin/streptomycin. C2C12 myoblasts were maintained in DMEM supplemented with 20\% FBS and switched to DMEM with 5\% horse serum (Invitrogen) when cell density reached confluency of $\sim 90 \%$. For AChR staining, C2C12 cells were grown on dishes coated with laminin (to promote the formation of large receptor clusters).

All transfections were performed using Lipofectamine 2000 (Invitrogen) as per the recommendation of the manufacturer with minor modifications. C2C12 transfection was performed in serum-free medium for $8 \mathrm{~h}$ before switching cells to the differentiation medium. HEK293T cells were either harvested for immunoblotting or fixed for confocal imaging $40-48 \mathrm{~h}$ after transfection.

Pharmacological agents. The proteasome inhibitor MG132 was purchased from Enzo Life Sciences and was used at $5 \mu \mathrm{M}$. Leupeptin, lactacystin, and chloroquine were purchased from Sigma-Aldrich and used at 20,10 , and $25 \mu \mathrm{g} / \mathrm{ml}$, respectively.

Acetylcholine chloride (Sigma) was made as a $1 \mathrm{~m}$ stock solution in distilled water and kept frozen. On the day of recording, it was diluted to the concentration of choice in extracellular recording solution.

$R T-P C R$. Total RNA was isolated from cultured myotubes using the RNAeasy Mini Kit (Qiagen). cDNA was synthesized using Superscript First Strand System and oligo-dT primers (Invitrogen). PCR was performed using the following primers: AChR $\alpha, 5^{\prime}$-ACGGCGACTTTGCC ATTGTC and 5' -CAGGCGCTGCATGACGAAGT; GAPDH, 5' -TGAA GGTCGGTGTGAACGGATTTGGC and 5'-CATGTAGGCCATGA GGTCCACCAC.
RT-PCR products were analyzed from samples collected in the exponential phase of amplification (35 cycles for AChR and 30 cycles for GAPDH).

Immunofluorescence and confocal microscopy. To assess AChR clustering, $\mathrm{C} 2 \mathrm{C} 12$ myoblasts grown on laminin-coated dishes were transfected with $4 \mu \mathrm{g}$ of pSuper.GFP expressing scrambled shRNA or shRNA( $\alpha$ kap). Eight hours after transfection, myoblasts were transferred into differentiation medium. Four to five days later, myotubes were bathed with $\alpha$-bungarotoxin ( $\alpha$-BTX) conjugated to Alexa Fluor-594 (BTX-Alexa594) (Invitrogen) at $4^{\circ} \mathrm{C}$ for $1 \mathrm{~h}$, washed in PBS, and then fixed in $2 \%$ paraformaldehyde (PFA). Cells were washed with PBS, mounted in DAPI Prolong Gold anti-fade medium (Invitrogen), and imaged with a Leica SP5 confocal microscope. The $z$-stacks were then collapsed, and the contrast was adjusted with Photoshop (Adobe Systems).

To compare the proportion of the myotube surface covered by AChR clusters under control conditions and when $\alpha$ kap was knocked down, we identified fields in dishes transfected with pSuper.GFP-shRNA $(\alpha$ kap $)$ in which there were both green fluorescent (transfected) and untransfected control myotubes and then used functions in NIH ImageJ (version 1.45i) to quantify the extent of clustering. We first applied a binary threshold to the entire red image (BTX-Alexa594), so that all regions with intensity below the threshold (background autofluorescence and fluorescence attributable to nonclustered receptors) were assigned to white, and the higher-intensity regions of clustered receptors were assigned to black. We then outlined at least one fluorescent and one nonfluorescent myotube on the green image (EGFP) and overlaid these regions of interest onto the thresholded images of AChR. We then applied the histogram function to each region of interest to get the number of pixels that were black or white. Finally, we calculated the proportion of each myotube covered by clustered receptors as (number of black pixels)/(total number of pixels in the region of interest).

To assess the localization of AChR $\alpha$ within HEK293T cells, cells were cotransfected with plasmids expressing GFP- $\alpha$ kap, $\mathrm{AChR} \alpha$, and pdiA3mCherry. Forty to forty-eight hours later, cells were washed with PBS, fixed in 4\% PFA for $10 \mathrm{~min}$ at room temperature, bathed in a blocking solution (10\% BSA) for $15 \mathrm{~min}$, and permeabilized with a solution containing $0.2 \%$ Triton X-100 and 2\% BSA in PBS for 15 min. Cells were incubated overnight with primary monoclonal anti-AChR $\alpha$ antibody MAB35 (The Developmental Studies Hybridoma Bank) diluted at 1:1000. Alexa Fluor-647-conjugated anti-mouse secondary antibody (Jackson ImmunoResearch) was added to cells for $1 \mathrm{~h}$. Coverslips were mounted onto glass slides using DAPI-Prolong Gold antifade medium (Invitrogen) and imaged at the appropriate wavelengths with a Leica SP5 confocal microscope.

Immunoprecipitation, pull down, and Western blotting. Transfected and untransfected C2C12 myotubes or HEK293T cells were lysed in buffer containing $50 \mathrm{~mm}$ HEPES, $300 \mathrm{~mm} \mathrm{NaCl}, 2$ mM EDTA, 0.5\% NP-40, 0.8\% CHAPS, $1 \mathrm{~mm}$ PMSF, $2 \mathrm{~mm}$ activated sodium orthovanadate, $5 \mathrm{~mm} \mathrm{NaF}$, and protease inhibitors. Lysates were incubated for $1 \mathrm{~h}$ at $4^{\circ} \mathrm{C}$ and cleared by centrifugation. Each lysate was assayed for anti-tubulin reactivity to assess the amount of protein present, and then aliquots of each lysate were typically used for several different procedures.

To selectively pull down surface AChR, living myotubes (untransfected or transfected with or without $\alpha$ kap shRNA) were incubated with BTX-biotin for $1 \mathrm{~h}$ at $4^{\circ} \mathrm{C}$. Cells were then lysed, and lysates were incubated with NeutrAvidin beads (Thermo Fisher Scientific) overnight. To selectively pull down intracellular AChR, living myotubes were bathed with unlabeled BTX to saturate all surface receptors for $1 \mathrm{~h}$ at $4^{\circ} \mathrm{C}$. Cells were then lysed, and lysates were incubated with BTX-biotin, followed by NeutrAvidin beads overnight. To pull down total AChR, cells were lysed with no pretreatment, and then lysates were incubated with BTXbiotin, followed by NeutrAvidin beads overnight. For all three conditions, the beads were collected by centrifugation and washed in PBS four times. The bound proteins were released by boiling in reducing $2 \times$ lithium dodecyl sulfate (LDS) buffer (Invitrogen).

To pull down CaMKIIs, muscle cell lysates were incubated overnight with calmodulin beads (Sigma) in the presence of $2 \mathrm{~mm} \mathrm{CaCl}_{2}$. The beads were collected and washed four times with buffer containing $0.5 \% \mathrm{NP}$ 40. Because $\alpha$ kap is known to bind to multiple CaMKII isoforms, this 
procedure was expected to pull down $\alpha$ kap, although it lacks a calmodulin binding domain.

The ProFound Mammalian HA-tag IP/Co-IP Kit (Thermo Fisher Scientific) was used to examine $\alpha$ kap and AChR $\alpha$ interaction in HEK293T cells following the instructions of the manufacturer in the presence of $300 \mathrm{mM}$ $\mathrm{NaCl}$ in the M-PER Mammalian Protein extraction reagent. The immunoprecipitate was then probed with anti-HA (Covance) or anti- $\alpha$ kap (Santa Cruz Biotechnology) and anti-AChR $\alpha$ (MAB210; Covance) antibodies.

To assay AChR ubiquitination, HEK293T cells were cotransfected with $\alpha$ kap, HA-tagged ubiquitin (HA-Ub), and either wild-type or mutated AChR $\alpha$ constructs (see above). The cells were harvested and lysed in denaturing buffer [ $50 \mathrm{~mm}$ Tris $\mathrm{HCl}, 150 \mathrm{~mm} \mathrm{NaCl}, 2 \mathrm{~mm}$ EDTA, $5 \mathrm{~mm}$ $\mathrm{N}$-ethylmaleimide (Sigma), $2 \mathrm{~mm}$ activated sodium orthovanadate, $2 \%$ SDS, and protease inhibitors], boiled for $10 \mathrm{~min}$, and briefly sonicated. The lysates were diluted to $0.1 \%$ SDS using buffer (50 mM HEPES, $\mathrm{pH}$ 7.4, $150 \mathrm{~mm} \mathrm{NaCl}, 2$ mм EDTA, 1\% NP-40, 1 mм PMSF, 2 mм activated sodium orthovanadate, $5 \mathrm{~mm} \mathrm{NaF}$, and protease inhibitors). Precleared protein lysates $(20 \mu \mathrm{g})$ were incubated with $2 \mu \mathrm{g}$ of anti-AChR $\alpha$ (MAB153; Santa Cruz Biotechnology) for $1 \mathrm{~h}$ at $4^{\circ} \mathrm{C}$, followed by additional incubation with $20 \mu \mathrm{l}$ of Protein A/G Plus-agarose beads (Santa Cruz Biotechnology) overnight at $4^{\circ} \mathrm{C}$. Beads were collected by centrifugation, extensively washed with lysis buffer without SDS, resuspended in LDS reducing sample buffer, and boiled for $5 \mathrm{~min}$. Proteins were separated by SDS-PAGE and transferred to PVDF membranes, which were then incubated in $10 \mathrm{~mm}$ Tris, $\mathrm{pH} 7.4,1 \mathrm{~mm}$ PMSF, $10 \mathrm{~mm}$ $\beta$-mercaptoethanol, and $6 \mathrm{M}$ guanidine for $1 \mathrm{~h}$ at $4^{\circ} \mathrm{C}$, followed by extensive washing in PBS. Membranes were then incubated with primary antibodies diluted in blocking buffer ( $2 \%$ fat-free milk in PBS) overnight at $4^{\circ} \mathrm{C}$. After extensive washing, the membranes were then incubated with secondary HRP-conjugated antibodies and developed using SuperSignal West Femto Maximum Sensitivity Substrate or West Pico (Thermo Fisher Scientific). Mouse anti-ubiquitin (Santa Cruz Biotechnology) and mouse anti-HA (clone A1; Covance) antibodies were diluted at 1:1000, mouse anti- $\alpha$ kap (clone A1; Santa Cruz Biotechnology) was diluted at 1:200, rabbit anti-CaMKII (M176; Santa Cruz Biotechnology) was diluted at 1:500, rabbit anti-GFP and rat anti-AChR $\alpha$ MAB210 (Covance) were diluted at 1:5000, mouse anti-tubulin (Developmental Studies Hybridoma Bank) was diluted 1:10,000, rabbit anti-Lrp4 was diluted 1:10,000, anti-LAMP1 was diluted 1:1000, and anti-P2X $\mathrm{X}_{2}$ was diluted 1:5000. All horseradish peroxidase-conjugated secondary antibodies (Jackson ImmunoResearch) were diluted at 1:10,000.

The intensity of bands on Western blots was quantified by scanning the film at 8 bits with white assigned a value of 0 and black a value of 255 . We then conducted the analysis using NIH ImageJ (version 1.45). We first verified that there was no saturation in the darkest regions of the blot and then selected a region of the film with no signal, which was used to calculate a background level that was subtracted from all pixel values so that the subtracted baseline was assigned a value of 0 . We then used the "magic wand" function to select the pixels of each band and the measure function to determine the integrated intensity of the selected region. This value is linearly proportional to the amount of reaction product imaged on the film but not necessarily to the amount of protein present. For all data presented as histograms, results from several blots were averaged, with the number indicated in the figure legend. The error bars represent the SD.

Electrophysiology. HEK293T cells were cotransfected with either all four wild-type AChR subunits $(\alpha, \beta, \delta$, and $\varepsilon$ ) or the $4 \mathrm{R}$ mutant of $\alpha$ and wild-type $\beta, \delta$, and $\varepsilon$. Each transfection mix also included a plasmid encoding EGFP.

Whole-cell recordings from green fluorescent cells were made $\sim 48 \mathrm{~h}$ after transfection, using a Dagan 3900 patch clamp and Clampex 9.0 software (Molecular Devices). All recordings were made at room temperature with the holding potential set to $-50 \mathrm{mV}$. The perfusion solution was switched from extracellular solution alone to extracellular solution plus acetylcholine with a BPS8 solution switcher (ALA). The cell was perfused by solutions applied from a narrow-bore pipette placed $\sim 1$ $\mathrm{mm}$ from the cell. The $10-90 \%$ exchange time of this system was $\sim 25 \mathrm{~ms}$.

The extracellular recording solution contained the following (in $\mathrm{mM}$ ): $135 \mathrm{NaCl}, 1.5 \mathrm{KCl}, 1 \mathrm{CaCl}_{2}, 1 \mathrm{MgCl}_{2}$, and 15 HEPES, pH 7.5. The intra-

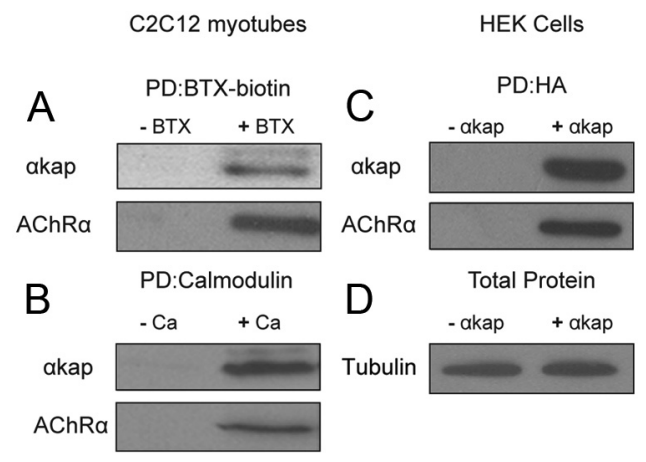

Figure 1. $\quad \alpha$ kap forms complexes with AChR in skeletal muscle cells. $A$, Lysates from cultured $\mathrm{C} 2 \mathrm{C} 12$ myotubes were incubated with $(+\mathrm{BTX})$ or without $(-\mathrm{BTX}) \mathrm{BTX}$-biotin, and then biotin containing complexes were isolated with NeutrAvidin beads. Pull-down proteins (PD) were probed with anti- $\alpha$ kap or anti-AChR $\alpha$ antibody. $B$, Myotube lysates were incubated with calmodulin beads in the absence of calcium $(-\mathrm{Ca}$ ) or in the presence $2 \mathrm{~mm}$ calcium $(+\mathrm{Ca})$ to pull down calcium/calmodulin binding proteins. Blots were probed with anti- $\alpha$ kap or antiAChR $\alpha$ antibody. C, Lysates from human embryonic kidney (HEK293T) cells transfected with AChR $\alpha$ and $\alpha$ kap-HA ( $+\alpha$ kap) or with AChR $\alpha$ and empty plasmid ( $-\alpha$ kap) were immunoprecipitated with anti-HA-coupled agarose beads, and blots were probed with anti- $\alpha$ kap or anti-AChR $\alpha$ antibodies. $\boldsymbol{D}$, Aliquots from the lysates used for the pull downs in $\mathbf{C}$ probed for tubulin. For all four panels, at least three independent experiments were performed.

cellular solution contained the following (in $\mathrm{mm}$ ): $140 \mathrm{KCl}, 1 \mathrm{MgCl}_{2}, 10$ EGTA, and 10 HEPES, pH 7.5.

\section{Results}

\section{Acetylcholine receptors form complexes with $\alpha$ kap}

Previous studies have shown that $\alpha$ kap is able to directly interact with many CaMKII isoforms (Bayer et al., 1996, 2002), and because CaMKII has been shown to control the density of AChR at the mature NMJ (Martinez-Pena y Valenzuela et al., 2010), we asked whether $\alpha$ kap could be involved in this process. To test this idea, lysates from cultured C2C12 myotubes were incubated with BTX-biotin to label AChR, and then AChR-BTX-biotin complexes were isolated with NeutrAvidincoated beads and subjected to Western blot analysis with antiAChR $\alpha$ and anti- $\alpha$ kap antibodies. $\alpha$ kap was pulled down with $\mathrm{AChR} \alpha$ (Fig. 1A), indicating that AChRs are present in the same complex as $\alpha$ kap. Similarly, when calmodulin-coated beads were used, we also pulled down $\operatorname{AChR} \alpha$ with $\alpha$ kap (Fig. $1 B)$. It should be noted that neither AChR nor $\alpha$ kap have calmodulin binding sites, indicating that the pull down by calmodulin beads is indirect, presumably because of the known ability of $\alpha$ kap to bind to multiple calmodulin-binding CaMKII isoforms. Thus, proteins besides $\mathrm{AChR} \alpha$ and $\alpha \mathrm{kap}$ are present in the material pulled down from cells that endogenously express both proteins. The interaction of $\alpha$ kap and AChR $\alpha$ was also observed when HEK293T cells (which lack endogenous $\alpha$ kap and AChR subunits) were cotransfected with $\mathrm{AChR} \alpha$ and HA-tagged $\alpha$ kap constructs (Fig. 1D). AChR $\alpha$ was not pulled down by anti-HA-coupled agarose beads when it was expressed alone, but both $\alpha$ kap and $\operatorname{AChR} \alpha$ were present in the lysate that was pulled down by anti-HA when cells were cotransfected. This result suggests that either $\alpha$ kap directly interacts with $\mathrm{AChR} \alpha$ or other components of the complex that link these two proteins are expressed in both muscle and HEK293T cells.

\section{Knockdown of $\alpha$ kap decreased AChR protein levels}

Having demonstrated that $\alpha$ kap is present in a complex with $\operatorname{AChR} \alpha$, we next tested whether changing the expression of $\alpha$ kap 
A
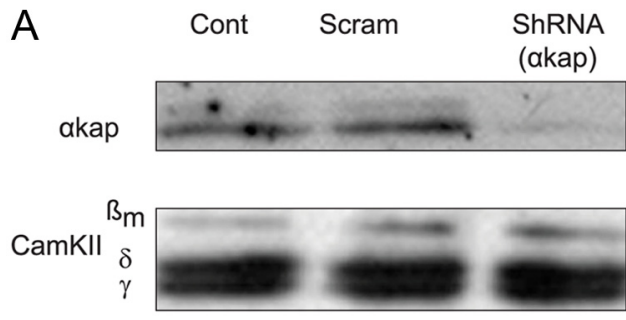

Tubulin

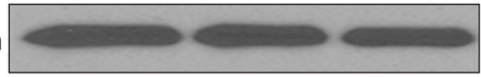

B

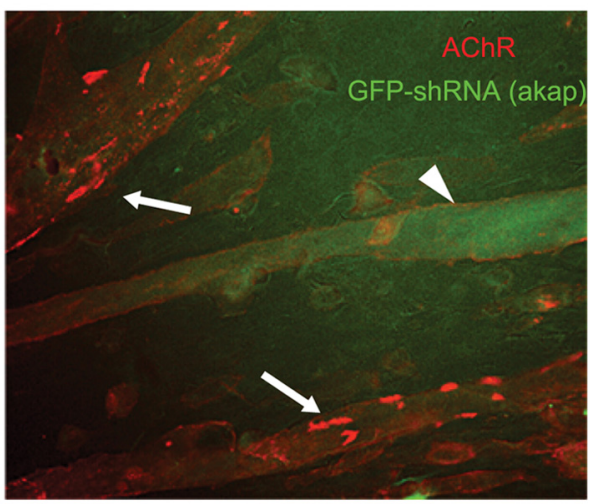

Figure 2. Knockdown of $\alpha$ kap significantly reduced the formation of AChR clusters on the surface of muscle cells. (2C12 myoblasts were transfected with either shRNA( $\alpha$ kap) or scrambled shRNA, and then 4-5 d after their differentiation, myotubes were either harvested for protein extraction and immunoblotting or bathed with BTX-Alexa594 to label surface AChR. A, Western blots of equal amount of proteins from untransfected myotubes (Cont), myotubes transfected with shRNA ( $\alpha$ kap) (a plasmid expressing shRNA specific to $\alpha$ kap), or a plasmid containing a scrambled version of the shRNA sequence (Scram). Blots were probed with anti$\alpha$ kap or anti-CaMKII (M176) antibody. M176 recognizes multiple CaMKII isoforms, including three that are expressed in skeletal muscle $\left(\beta_{\mathrm{m}}, \delta\right.$, and $\left.\gamma\right)$. Tubulin was used as loading control. $B$, Untransfected myotubes (arrows) and a myotube transfected with GFP-shRNA( $\alpha$ kap) viewed in the same dish to allow comparison of the number of AChR clusters when $\alpha$ kap is knocked down. Very few clustered receptors were present on transfected (green fluorescent, arrowhead) myotubes.

had an effect on the clustering of AChR on the muscle cell surface. To do this, we used $\alpha$ kap-specific shRNA to knock down the expression of $\alpha \mathrm{kap}$ in $\mathrm{C} 2 \mathrm{C} 12$ muscle cells. We designed two different shRNAs (shRNA137 and shRNA337), and as an initial test of the efficiency of the knock down, we transfected C2C12 myoblasts with GFP- $\alpha$ kap alone or cotransfected with GFP- $\alpha$ kap and either shRNA137 or shRNA337. When myotubes were viewed $4-5 \mathrm{~d}$ later, there were many green fluorescent fibers in the dishes transfected with GFP- $\alpha$ kap alone but very few fluorescent fibers in the dishes cotransfected with GFP- $\alpha$ kap and shRNAs (data not shown), indicating that both shRNAs were effective in knocking down the expression of exogenous $\alpha$ kapGFP. Western blot analysis of equal amount of proteins with anti- $\alpha$ kap antibody showed that transfection with either shRNA drastically reduced the endogenous expression of $\alpha$ kap protein compared with untransfected myotubes or myotubes transfected with a scrambled shRNA (Fig. $2 A$ ). In contrast, neither of the shRNAs had an effect on the expression level of three CaMKII isoforms $\left(\beta_{\mathrm{m}}, \gamma\right.$, and $\left.\delta\right)$ that have several domains similar to domains of $\alpha$ kap (Fig. $2 A$ ). We performed all remaining experiments using shRNA337, which will be referred to as $\operatorname{shRNA}(\alpha \mathrm{kap})$.
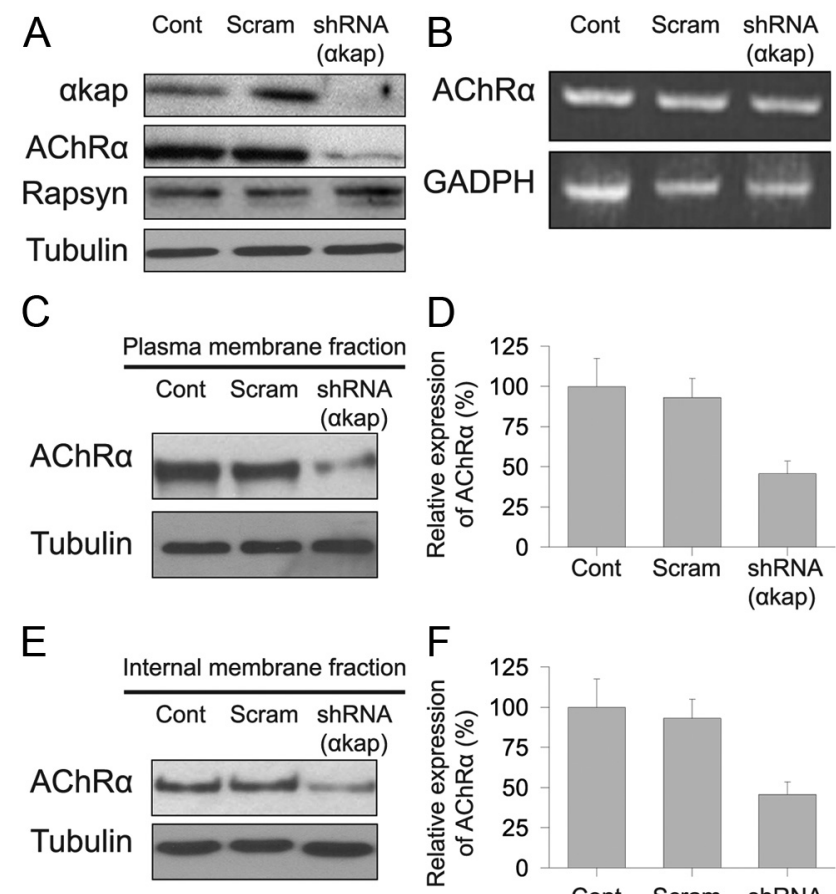
(akap)

Figure 3. Knockdown of $\alpha$ kap reduced AChR protein levels. $\boldsymbol{A}$, Equal amounts of proteins from lysates of myotubes transfected with shRNA( $\alpha$ kap) or scrambled shRNA (Scram) or untransfected myotubes (Cont) were probed with four antibodies to assess the effect of the shRNA on $\alpha$ kap, AChR $\alpha$, rapsyn, and tubulin. $B$, RT-PCR products of AChR $\alpha$ from cultured myotubes transfected with shRNA $(\alpha$ kap), scrambled shRNA, and untransfected myotubes. The PCR reaction conditions did not allow saturation to be reached for any of the samples, so band intensity is proportional to the amount of mRNA in the original sample. $\boldsymbol{C}$, Western blots of surface $\mathrm{AChR} \alpha$. Living myotubes were treated with BTX-biotin at $4^{\circ} \mathrm{C}$ to saturate all surface receptors before cell lysis. Biotin-labeled surface $A C h R s$ were precipitated with NeutrAvidin beads. AChR $\alpha$ subunits were detected with MAB210. D, Quantification of three blots as in C. E, Western blots of intracellular $A C h R \alpha$. Surface receptors were saturated with unlabeled BTX, and then muscle lysates were saturated with BTX- biotin to selectively label intracellular AChR. F, Quantification of three blots as in $\boldsymbol{E}$.

Next we examined the effect of the knockdown of $\alpha$ kap on AChR clusters on the surface of muscle cells. C2C12 myoblasts were cotransfected with plasmids encoding EGFP and either $\operatorname{shRNA}(\alpha$ kap $)$ or its scrambled version and grown on laminincoated dishes. The AChR clusters (identified by their labeling with fluorescent BTX) were fewer and smaller on myotubes transfected with shRNA (identified by their labeling with EGFP) compared with either untransfected myotubes in the same dish (Fig. 2B) or to myotubes in separate dishes transfected with the scrambled shRNA alone (data not shown). The average percentage of the surface covered by clustered receptors in control myotubes was $6.8 \pm 3.3 \%$, but this fell to $0.7 \pm 0.2 \%$ in $\operatorname{shRNA}(\alpha$ kap $)$ knockdown myotubes ( \pm SEM, $n=10$ fibers each).

The decrease in the number of clusters of AChR on the surface of myotubes transfected with shRNA could have been produced by several mechanisms. The morphology of cultured myotubes cotransfected with EGFP and $\operatorname{shRNA}(\alpha$ kap) plasmids was indistinguishable from untransfected myotubes, indicating that $\alpha$ kap knockdown had no effect on the differentiation of myoblasts into myotubes. Because AChR forms a complex with several other postsynaptic proteins, another possibility was that depletion of $\alpha$ kap might lead to a decrease in other components of the complex that are involved in receptor clustering. However, Western blot results showed that $\operatorname{shRNA}(\alpha \mathrm{kap})$ produced a dramatic de- 

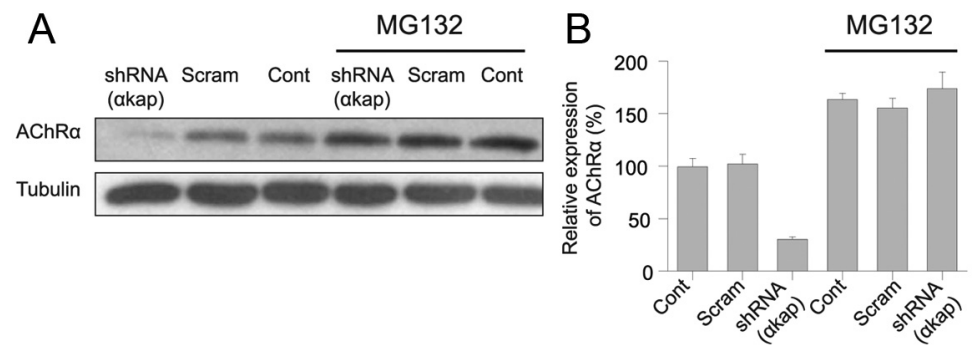

C

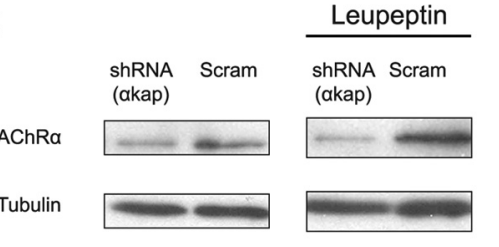

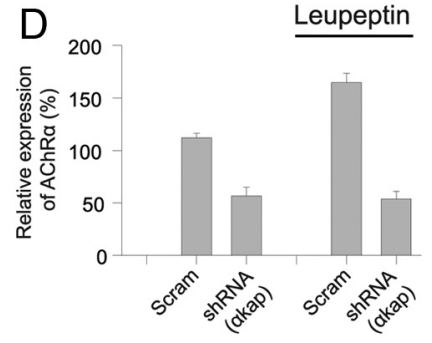

Figure 4. A proteasome inhibitor prevented AChR degradation in cells transfected with shRNA( $\alpha$ kap). $\boldsymbol{A}$, Myotubes transfected with shRNA $(\alpha k a p)$, scrambled shRNA (Scram), or untransfected myotubes (Cont) were treated with the proteasome inhibitor MG132 (at $5 \mu \mathrm{M}$ ), and lysates were immunoblotted with MAB210 antibody against AChR $\alpha$. Tubulin was used as a loading control. $B$, Quantification of three independent experiments as in $\boldsymbol{A} .100 \%$ was the average intensity of the samples from untransfected myotubes in the absence of MG132. C, Myotubes transfected with plasmids encoding shRNA( $\alpha$ kap) or the scrambled shRNA were treated with leupeptin $(20 \mu \mathrm{g} / \mathrm{ml})$. D, Quantification of three independent experiments as in C. $100 \%$ was the average intensity of the samples from myotubes transfected with the scrambled shRNA in the absence of leupeptin. crease in $\mathrm{AChR} \alpha$ but no change in the level of the AChR clustering protein rapsyn (Fig. $3 A$ ). We next tested whether the decrease in the amount of AChR $\alpha$ was attributable to a decrease in AChR mRNAs. However, when RT-PCR products specific to AChR $\alpha$ were analyzed (Fig. 3B), we found that there was no difference in the amount of $\mathrm{AChR} \alpha$ mRNAs between untransfected and $\alpha$ kapdepleted myotubes. If $\alpha$ kap is involved specifically in clustering AChR on the surface of myotubes, the amount of AChR on the plasma membrane should be the same in myotubes transfected with $\operatorname{shRNA}(\alpha \mathrm{kap})$ and scrambled shRNA and in untransfected myotubes. However, in myotubes transfected with $\operatorname{shRNA}(\alpha \mathrm{kap})$, the amount of surface AChR $\alpha$ was dramatically reduced compared with scrambled or untreated myotubes (Fig. 3C,D). We also found that fewer receptors were detected in the internal pool of myotubes transfected with shRNA( $\alpha$ kap) compared with myotubes transfected with scrambled shRNA or to control myotubes (Fig. 3E,F). Thus, both the intracellular and the surface pools of AChR $\alpha$ were depleted by similar amounts.

\section{akap controls AChR degradation}

Having shown that knockdown of $\alpha$ kap has no effect on mRNA levels for $\mathrm{AChR} \alpha$ but drastically reduced $\mathrm{AChR} \alpha$ protein levels, we hypothesized that $\alpha$ kap might control the stability of AChR. As an initial test, muscle cells were treated with MG132 (carbobenzoxy-L-leucyl-L-leucyl-L-leucinal), a widely used proteasome inhibitor that can prevent degradation of many ERassociated degradation (ERAD) substrates (Rock et al., 1994) or with leupeptin, a lysosome inhibitor. We found that, in untransfected myotubes or myotubes transfected with the scrambled shRNA, both MG132 and leupeptin increased the amount of AChR $\alpha$ protein to $\sim 160 \%$ of the untreated control (Fig. 4), indicating that both inhibitors were able to prevent $\mathrm{AChR} \alpha$ degradation. However, the two inhibitors had quite different effects on C2C12 myotubes that had been transfected with shRNA( $\alpha \mathrm{kap})$. The effect of MG132 on cells transfected with $\operatorname{shRNA}(\alpha$ kap) was quite similar to the effect of MG132 on untransfected cells (Fig.
$4 A, B)$, whereas the effect of leupeptin plus $\operatorname{shRNA}(\alpha$ kap) was quite similar to shRNA $(\alpha$ kap) alone (Fig. $4 C, D)$. Thus, in the presence of $\operatorname{shRNA}(\alpha \mathrm{kap})$, MG132 produced an average increase of $578 \pm$ $16 \%$ in $\operatorname{AChR} \alpha$, whereas leupeptin produced no change (95 $\pm 8 \%$ ). A second pair of inhibitors showed similar trends. In the presence of $\operatorname{shRNA}(\alpha \mathrm{kap})$, the proteasome inhibitor lactacystin produced a significant increase in $\mathrm{AChR} \alpha$ (average of $333 \pm 62 \%, p<0.05, n=4)$, but the lysosomal inhibitor chloroquine produced no significant increase in $\mathrm{AChR} \alpha$ (average of $161 \pm 47 \%, p>0.7)$. These results suggest that $\alpha$ kap protects AChR from degradation by the proteasome but not from degradation within lysosomes.

To better understand the mechanism by which $\alpha$ kap controls the amount of AChR in myotubes, we used heterologous expression in HEK293T cells, which lack endogenous $\alpha$ kap and $\operatorname{AChR} \alpha$. First we tested whether $\alpha$ kap promotes the stability of AChR $\alpha$ in HEK293T cells, as it does in cultured myotubes. Lysates from cells transfected with EGFP and $\operatorname{AChR} \alpha$ with or without $\alpha$ kap were immunoblotted with anti-AChR $\alpha$ antibody, and AChR $\alpha$ levels were compared. The amount of AChR $\alpha$ was nearly threefold higher in cells cotransfected with $\alpha$ kap compared with cells transfected with only $\operatorname{AChR} \alpha$, indicating that $\alpha$ kap promotes the stability of AChR in HEK293T cells (Fig. $5 A, C)$. Treatment of HEK293T cells expressing AChR $\alpha$ and a control plasmid with MG132 also increased $\mathrm{AChR} \alpha$ levels by approximately threefold compared with untreated control cells (Fig. 5A-C). However, the combined effect of $\alpha$ kap and MG132 on $\operatorname{AChR} \alpha$ accumulation was no larger than either treatment alone, indicating that $\alpha$ kap is sufficient to maximally protect $\mathrm{AChR} \alpha$ from degradation by the proteasome (Fig. 5C).

Next, we tested whether $\alpha$ kap is involved in controlling the stability of other AChR-associated components expressed in HEK293T cells. In contrast to $\operatorname{AChR} \alpha$, the amount of $\alpha$-syntrophin or $\alpha$-dystrobrevin (two components of the postsynaptic dystrophin glycoprotein complex that tethers AChR) was similar in the presence or absence of $\alpha$ kap (Fig. 5D). However, like $\mathrm{AChR} \alpha$, the amount of Lrp4, a transmembrane protein that transmits a signal required for clustering of AChRs, was significantly higher in cells cotransfected with $\alpha \mathrm{kap}$ ( $190 \pm 10 \%$ of the level in cells transfected with Lrp 4 only, $n=4$; Fig. $5 E$ ). To explore the possibility that $\alpha$ kap might be acting as a general regulator of the stability of transmembrane proteins, we tested two additional transmembrane proteins expressed in HEK293T cells. Neither rat P2X 2 (a plasma membrane protein) nor LAMP1 (a lysosomal membrane protein) was sensitive to the presence of $\alpha$ kap (Fig. 5E). Thus, $\alpha$ kap is not a general regulator of transmembrane proteins.

Previous studies have shown that, in several cell types, $\alpha$ kap is primarily localized to the ER compartment (Nori et al., 2003; Singh et al., 2009) and that, when $\operatorname{AChR} \alpha$ is expressed without other AChR subunits, it remains mostly trapped in the ER (Christianson and Green, 2004). We therefore examined whether AChR $\alpha$ colocalizes with $\alpha$ kap in the ER of HEK293T cells. Confocal microscopy analysis of cells cotransfected with monomeric 
A
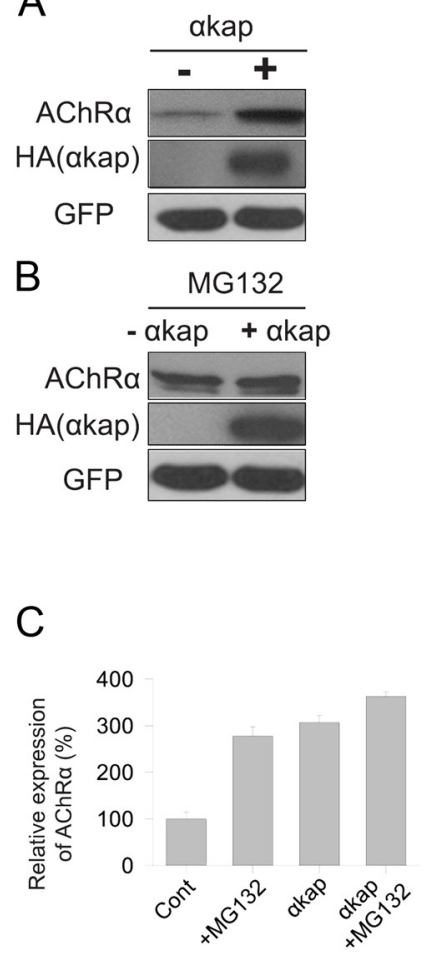

$\mathrm{F}$

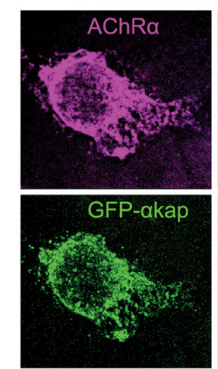

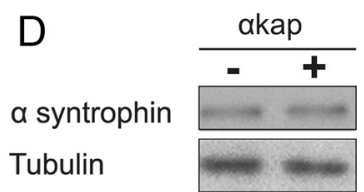

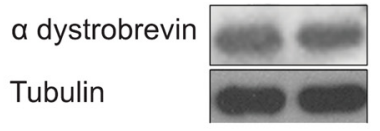

$E$
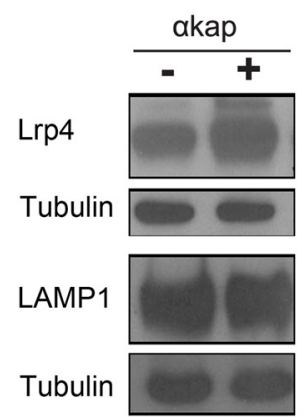

P2X2

Tubulin

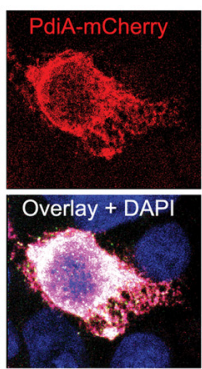

Figure 5. $\quad \alpha$ kap increased AChR $\alpha$ accumulation in transfected HEK cells. $A$, Western blots of homogenates from HEK293T cells cotransfected with $\mathrm{AChR} \alpha$, HA- $\alpha$ kap and pEGFP-C3(+) constructs or with $\mathrm{AChR} \alpha$, pEGFP-C3 constructs, and an empty version of the same vector into which $\alpha$ kap-HA was inserted (-). GFP was used as a control for the transfection efficiency and for the loading of total proteins. Anti-HA antibody was used to confirm the expression of $\alpha$ kap. $B$, Experiment identical to $A$, except that MG132 was present. C, Quantification of two independent replicates of experiments as in $\boldsymbol{A}$ and $\boldsymbol{B}$. D. Western blots of lysates from HEK293T cells transfected with $\alpha$-syntrophin or $\alpha$-dystrobrevin with or without $\alpha$ kap. $E$, Lysates from HEK293T cells transfected with Lrp4 or P2X with or without $\alpha$ kap were probed with antibodies against Lrp4, P2X 2 , and LAMP1. F, Confocal images of an HEK293T cell coexpressing GFP- $\alpha$ kap, AChR $\alpha$, and pdiA-mCherry (a marker of the ER). The nucleus was labeled with DAPI. Cells were fixed, permeabilized, and stained with anti-AChR antibody (MAB35), followed by anti-rat Alexa Fluor-647-labeled secondary antibody to visualize AChR. pdiA and $\alpha$ kap were visualized by the fused fluorescent proteins.

GFP- $\alpha$ kap, AChR $\alpha$, and the ER marker mCherry-pdiA3 showed that they all reside in the ER under these conditions (Fig. 5E).

Because MG132 does not promote any additional AChR accumulation when $\alpha$ kap is present, we hypothesized that $\alpha$ kap might prevent ubiquitination of $\mathrm{AChR} \alpha$ and thus degradation. As a first test of this hypothesis, we cotransfected HEK293T cells with AChR $\alpha$ and HA-Ub (Fang and Kerppola, 2004) in the presence or absence of $\alpha$ kap and estimated the amount of AChR $\alpha$ in each sample by Western blots (Fig. $6 A$ ). We then used this information to equalize the amount of AChR $\alpha$ (Fig. $6 B$ ), which was then immunoprecipitated with $\mathrm{AChR} \alpha$ antibody and probed on an immunoblot with anti-ubiquitin antibody. The blots showed a

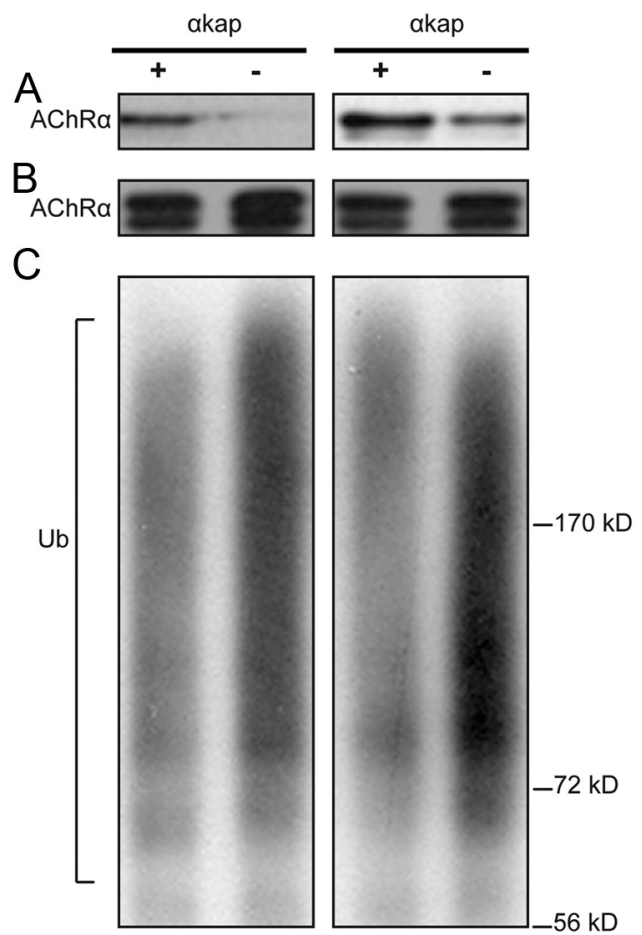

Figure 6. $\alpha$ kap inhibits ubiquitination of $\mathrm{AChR} \alpha$. Two independent replicates are shown in all three panels. $A$, The level of expression of AChR $\alpha$ in lysates from HEK293T cells cotransfected with $\mathrm{AChR} \alpha, \mathrm{HA}-\mathrm{Ub}$, and either a plasmid encoding $\alpha$ kap $(+)$ or empty vector $(-)$ as a control. Tubulin staining (data not shown) indicated that equal amounts of protein were loaded in the two lanes. $B, C$, Lysates with equal amounts of proteins were immunoprecipitated with anti-AChR $\alpha$ antibody (MAB153) and probed with AChR $\alpha$ antibody MAB210 (B) or with antiubiquitin antibody ( $($ ). To allow the amount of ubiquitination to be easily compared between experimental conditions, before the immunoprecipitation step the concentration of total proteins in each sample was adjusted so that similar amounts of $A C h R \alpha$ would be precipitated (see $\boldsymbol{B}$ ) although the amounts of AChR in the original samples were quite different (see $\boldsymbol{A})$. The material recognized by anti-ubiquitin appears as a smear because the extent of polyubiquitination varies widely.

smear, representing the addition of several ubiquitin moieties to the various lysine residues within $\mathrm{AChR} \alpha$ (Fig. 6C). By quantifying the intensity of the staining in the smear, we found that, when AChR was expressed alone, the level of $\mathrm{AChR} \alpha$ ubiquitination was substantially higher $(210 \pm 10 \%)$ than when it was coexpressed with $\alpha$ kap.

There are four lysines (K359, K374, K388, and K393) within the major intracellular loop of $\mathrm{AChR} \alpha$ that are predicted to be ubiquitinated (Radivojac et al., 2010). If the absence of $\alpha$ kap promotes ubiquitination of lysine residues on $\mathrm{AChR} \alpha$ leading to AChR degradation, changing lysine to arginine residues (so ubiquitin cannot be conjugated) should lead to less degradation of $\mathrm{AChR} \alpha$. To directly test this idea, we used site-directed mutagenesis to make four single mutant constructs (K359R, K374R, K388R, or K393R, 1R), a construct that contained three mutations $(\mathrm{K} 359 \mathrm{R} / \mathrm{K} 388 \mathrm{R} / \mathrm{K} 393 \mathrm{R}, 3 \mathrm{R})$, and a construct that had all four mutations (K359R/K374R/K388R/K393R, 4R). When coexpressed with HA-Ub in HEK293T cells, the 3R and 4R AChR $\alpha$ mutants exhibited a large decrease in ubiquitination compared with the wild-type AChR $\alpha$ or any of the four 1R mutants (Fig. 7A; data for only one of the $1 \mathrm{R}$ mutants is shown). The $4 \mathrm{R}$ mutant $\mathrm{AChR} \alpha$ was capable of assembling into a functional AChR complex when coexpressed with $\beta, \delta$, and $\varepsilon$ subunits (Fig. $7 B$ ). However, we decided not to pursue an electrophysiological approach to assess expression levels, because of the highly variable extent of 


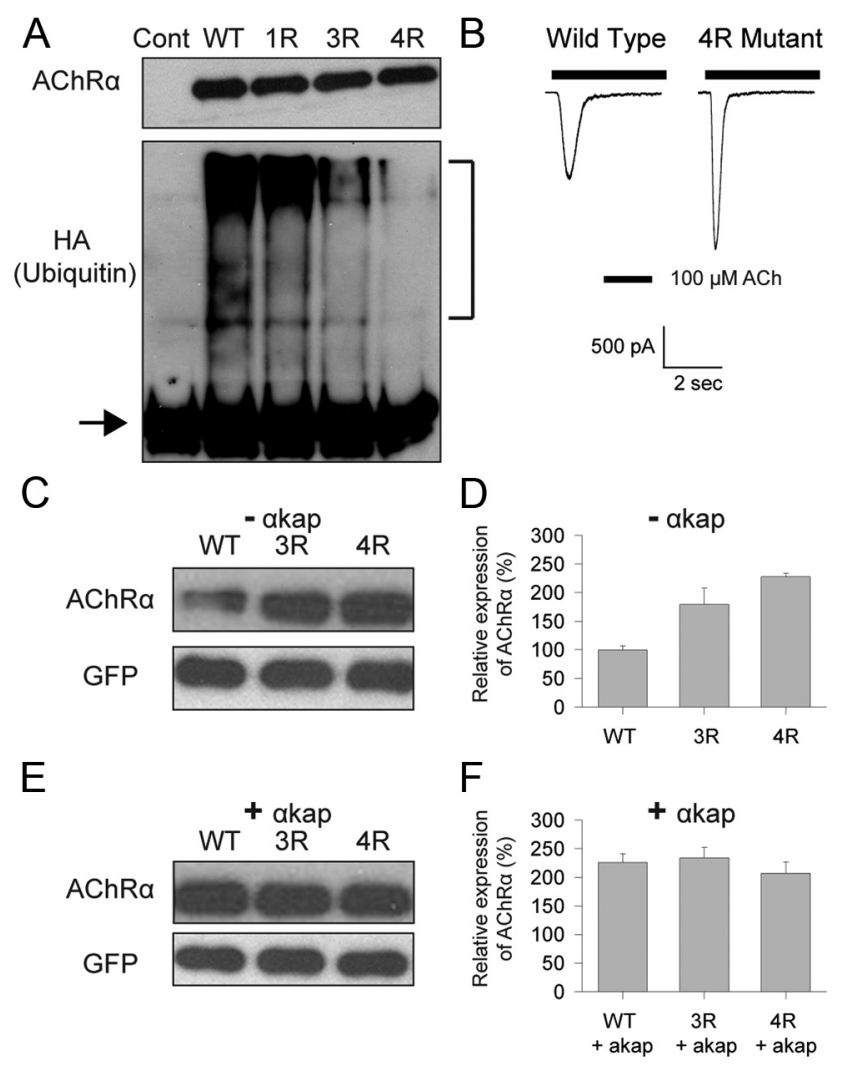

Figure 7. $\alpha$ kap had no effect on the stability of AChR $\alpha$ when lysines essential for ubiquitination were mutated. HEK293T cells were cotransfected with plasmids encoding the indicated $\mathrm{AChR} \alpha$ variants and $\mathrm{HA}-\mathrm{Ub}$, and $48 \mathrm{~h}$ later protein lysates were collected. The anti-AChR $\alpha$ antibody MAB153 was used to selectively immunoprecipitate proteins from each sample. $A$, The extent of ubiquitination of $\mathrm{AChR} \alpha$ detected with an anti-HA antibody. In this experiment, the amount of material loaded onto each lane was adjusted so that comparable amounts of $\mathrm{AChR} \alpha$ were present in all samples. The top gel shows the amount of $\mathrm{AChR} \alpha$ detected with anti-AChR $\alpha$ (MAB210), and the bottom gel shows the anti-HA immunoreactivity. The control lane (Cont) was from cells transfected with HA-Ub only. WT, Wild type. The 1R lane is the K359R mutant, the $3 \mathrm{R}$ lane is the $\mathrm{K} 359 \mathrm{R} / \mathrm{K} 388 \mathrm{R} / \mathrm{K} 393 \mathrm{R}$ mutant, and the $4 \mathrm{R}$ lane is the $\mathrm{K} 359 \mathrm{R} / \mathrm{K} 374 \mathrm{R} / \mathrm{K} 388 \mathrm{R} /$ K393R mutant. The lower band present in all lanes of the anti-HA blot (arrow) is the $25 \mathrm{kDa}$ light chain of the antibody used for immunoprecipitation and served as a loading control. The smear present in some lanes (brackets, $\sim 70-250 \mathrm{kDa}$ ) is polyubiquinated $\mathrm{AChR} \alpha$ and so is absent in the control because no $A C h R$ were present. Polyubiquitination of $A C h R \alpha$ was greatly decreased in the 3R and 4R mutants. B, Whole-cell recordings from HEK293T cells cotransfected with EGFP, wild-type $A C h R \beta, \delta$, and $\varepsilon$ subunits, and either the wild-type or the $4 R$ mutant $A C h R \alpha$. The holding potential was $-50 \mathrm{mV}$. ACh was applied for the duration indicated by the bar above each trace. C, Western blots of homogenates from HEK293T cells cotransfected with EGFP and wild-type $A C h R \alpha$ or the ubiquitination-deficient $3 R$ or $4 R$ mutant. In these experiments, the same amount of material (as determined from anti-GFP staining) was loaded onto each lane. № $\alpha$ kap was present $(-\alpha$ kap). D, Quantification of three independent experiments similar to $\boldsymbol{C}$. In this panel as well as $\boldsymbol{F}, 100 \%$ represents the intensity of signal produced when cells were transfected with wild-type AChR $\alpha$ in the absence of $\alpha$ kap. $\boldsymbol{E}$, Experiment similar to $\boldsymbol{C}$, except that the cells were also cotransfected with a plasmid encoding $\alpha$ kap ( $+\alpha$ kap). $\boldsymbol{F}$, Quantification of three experiments similar to $\boldsymbol{E}$.

transfection and because the rapid desensitization to maximal concentrations of ACh made the amplitude of the peak currents dependent on the position of the drug application pipette and the rate of flow out of it, as well as on the number of receptors present. When a biochemical approach was used to assess expression levels, the reduced levels of ubiquitination in the $3 \mathrm{R}$ and $4 \mathrm{R}$ mutants correlated with a significant increase in the stability of $\mathrm{AChR} \alpha$ mutants compared with wild-type (Fig. 7C,D). These results demonstrate that lysines $359,374,388$, and 393 represent major sites for ubiquitination and that ubiquitinating several of these lysines promotes degradation. The presence of $\alpha \mathrm{kap}$ did not increase the amount of $3 \mathrm{R}$ or $4 \mathrm{R}$ mutants compared with wild-type $\mathrm{AChR} \alpha$ (Fig. $7 E, F$ ), suggesting that the effect of $\alpha$ kap on AChR $\alpha$ stability is mediated by controlling AChR $\alpha$ ubiquitination.

\section{Discussion}

In this work, we provide evidence for a novel function of $\alpha$ kap in controlling AChR stability. We demonstrated the following: (1) loss of $\alpha$ kap drastically reduced AChR levels, leading to fewer receptor clusters on the surface of muscle cells; (2) two proteasome inhibitors prevented degradation of AChR $\alpha$ in the absence of $\alpha \mathrm{kap}$; (3) proteasome inhibition does not enhance $\mathrm{AChR} \alpha$ accumulation any further in the presence of $\alpha \mathrm{kap}$; (4) the absence of $\alpha$ kap increased ubiquitination of $\mathrm{AChR} \alpha$; (5) major sites for lysine ubiquitination reside within the intracellular domain of $\mathrm{AChR} \alpha$; and finally, (6) mutations of $\mathrm{AChR} \alpha$ lysine residues required for ubiquitination slow the degradation of $\operatorname{AChR} \alpha$.

Previous studies have shown that, in heterologous cells transfected with all receptor subunits, unassembled AChR subunits are rapidly degraded in the ER and that assembly into a pentameric complex is essential for protein stability (Claudio et al., 1989; Blount and Merlie, 1990). This degradation is thought to occur in the ERAD pathway in which ubiquitination and the proteasome are involved, because unassembled subunits were accumulated in cells treated with proteasome inhibitors (Christianson and Green, 2004). In the present study, we demonstrated that overexpression of $\alpha$ kap substantially increased the amount of unassembled $\mathrm{AChR} \alpha$ in the ER of HEK cells, even when the proteasome was functioning normally. In skeletal and cardiac muscles, $\alpha$ kap is highly expressed and localized to the sarcoplasmic reticulum (Nori et al., 2003; Singh et al., 2009). It was established previously that $\alpha$ kap associates with multiple CaMKII isoforms and anchors them to the sarcoplasmic reticulum through its hydrophobic domain (Campbell and MacLennan, 1982; Damiani et al., 1995; Bayer et al., 1998). This has the functional consequence of modulating $\mathrm{Ca}^{2+}$ release from the sarcoplasmic reticulum and so regulating excitation/contraction coupling in muscle cells (Bers, 2002a,b). Our work indicates that $\alpha \mathrm{kap}$ has a second important function as a regulator of AChR stability in muscle cells. The action of $\alpha$ kap on protein stability was not unique to AChR, because at least one additional muscle protein (Lrp4) was also $\alpha$ kap sensitive. However, two other transmembrane proteins (P2X2 and LAMP1) and several intracellular synaptic proteins (rapsyn, $\alpha$-dystrobrevin, and $\alpha$-syntrophin) were $\alpha$ kap insensitive. Thus, it is possible that modulation of $\alpha$ kap activity in vivo might have relatively selective actions on synaptic function.

The present experiments show that $\alpha$ kap interacts with $\mathrm{AChR} \alpha$ and promotes their stability and that the effect of $\alpha$ kap can be mimicked by elimination of ubiquitin conjugating sites from $\operatorname{AChR} \alpha$. How could $\alpha$ kap be linked to AChR stability? One possible molecular mechanism would be an indirect effect, with $\alpha$ kap acting as a chaperone to promote the forward trafficking of the AChR to the Golgi so that the receptors are not in the ER long enough to be modified. A second possibility is that $\alpha$ kap more directly regulates the state of ubiquitination of $\mathrm{AChR} \alpha$ in the ER. Our results favor the latter possibility, because in HEK cells expressing $\mathrm{AChR} \alpha$ alone, there is no movement to the Golgi and yet $\alpha$ kap still is effective. As far as how $\alpha$ kap might act on AChR within the ER, one possibility is that $\alpha$ kap may act as a deubiq- 
uitinase. This seems unlikely because $\alpha$ kap does not share any similarities with known deubiquitinases. A related possibility is that $\alpha$ kap may control AChR deubiquitination through an association with deubiquitinase(s). It has been reported that the ubiquitin-specific protease 19 (USP19) localizes in the ER and is involved in processing ERAD substrates (Hassink et al., 2009). Interestingly, Usp19 also interacts with CaMKII (Sowa et al., 2009), and, because $\alpha$ kap forms complexes with CaMKII, it is possible that $\alpha$ kap may function as a scaffold for Usp19. Other mechanisms by which $\alpha$ kap could decrease ubiquitination of AChR would be by inhibiting the E3 ubiquitin ligase(s) that conjugate ubiquitin to lysines on the receptors or by masking the lysines of $\mathrm{AChR} \alpha$ so they cannot be ubiquitinated.

Recent studies have shown that the ubiquitin-proteasome system is involved in regulating synaptic protein turnover and synaptic efficacy at a variety of synapses (Ehlers, 2003; Speese et al., 2003; Christianson and Green, 2004; Mabb and Ehlers, 2010). Ubiquitination has been shown to play a key regulatory mechanism for many cell-surface proteins, in addition to its classical function of targeting cytosolic proteins for degradation by the proteasome. For example, ubiquitination of AMPA receptors, NMDA receptors, and PSD scaffold proteins has emerged as the major mechanism for controlling the accelerated turnover and decreased amounts of receptor proteins and thus synaptic plasticity (Juo and Kaplan, 2004; Mabb and Ehlers, 2010). At the fly $\mathrm{NMJ}$, a balance between ubiquitination and deubiquitination plays a key role in shaping the morphology of the synapse. Loss of function of the USP highwire or its ortholog in C. elegans ( $r p m-1)$ produces profound defects in synaptic transmission (Schaefer et al., 2000; Wan et al., 2000). At developing mammalian NMJ, ubiquitination of the receptor tyrosine kinase MuSK leads to a reduction in postsynaptic MuSK expression levels and consequently defective synapses ( $\mathrm{Lu}$ et al., 2007). Our results indicate that an additional mechanism by which ubiquitination regulates receptor trafficking in an $\alpha$ kap-dependent manner is critical for maintaining the steady state of the postsynaptic receptor density and thus synaptic strength.

\section{References}

Adams ME, Kramarcy N, Krall SP, Rossi SG, Rotundo RL, Sealock R, Froehner SC (2000) Absence of alpha-syntrophin leads to structurally aberrant neuromuscular synapses deficient in utrophin. J Cell Biol 150:1385-1398.

Bayer KU, Löhler J, Harbers K (1996) An alternative, nonkinase product of the brain-specifically expressed $\mathrm{Ca}^{2+} /$ calmodulin-dependent kinase II alpha isoform gene in skeletal muscle. Mol Cell Biol 16:29-36.

Bayer KU, Harbers K, Schulman H (1998) alphaKAP is an anchoring protein for a novel CaM kinase II isoform in skeletal muscle. EMBO J 17:5598-5605.

Bayer KU, De Koninck P, Schulman H (2002) Alternative splicing modulates the frequency-dependent response of CaMKII to $\mathrm{Ca}^{2+}$ oscillations. EMBO J 21:3590-3597.

Bers DM (2002a) Cardiac excitation-contraction coupling. Nature 415:198-205.

Bers DM (2002b) Sarcoplasmic reticulum Ca release in intact ventricular myocytes. Front Biosci 7:d1697-d1711.

Blount P, Merlie JP (1990) Mutational analysis of muscle nicotinic acetylcholine receptor subunit assembly. J Cell Biol 111:2613-2622.

Campbell KP, MacLennan DH (1982) A calmodulin-dependent protein kinase system from skeletal muscle sarcoplasmic reticulum. Phosphorylation of a 60,000-dalton protein. J Biol Chem 257:1238-1246.

Christianson JC, Green WN (2004) Regulation of nicotinic receptor expression by the ubiquitin-proteasome system. EMBO J 23:4156-4165.

Chu A, Sumbilla C, Inesi G, Jay SD, Campbell KP (1990) Specific association of calmodulin-dependent protein kinase and related substrates with the junctional sarcoplasmic reticulum of skeletal muscle. Biochemistry 29:5899-5905.
Claudio T, Paulson HL, Green WN, Ross AF, Hartman DS, Hayden D (1989) Fibroblasts transfected with Torpedo acetylcholine receptor beta-, gamma-, and delta-subunit cDNAs express functional receptors when infected with a retroviral alpha recombinant. J Cell Biol 108:2277-2290.

Damiani E, Picello E, Saggin L, Margreth A (1995) Identification of triadin and of histidine-rich $\mathrm{Ca}^{2+}$-binding protein as substrates of $60 \mathrm{kDa}$ calmodulin-dependent protein kinase in junctional terminal cisternae of sarcoplasmic reticulum of rabbit fast muscle. Biochem Biophys Res Commun 209:457-465.

DeChiara TM, Bowen DC, Valenzuela DM, Simmons MV, Poueymirou WT, Thomas S, Kinetz E, Compton DL, Rojas E, Park JS, Smith C, DiStefano PS, Glass DJ, Burden SJ, Yancopoulos GD (1996) The receptor tyrosine kinase MuSK is required for neuromuscular junction formation in vivo. Cell 85:501-512.

Ehlers MD (2003) Activity level controls postsynaptic composition and signaling via the ubiquitin-proteasome system. Nat Neurosci 6:231-242.

Fang D, Kerppola TK (2004) Ubiquitin-mediated fluorescence complementation reveals that Jun ubiquitinated by Itch/AIP4 is localized to lysosomes. Proc Natl Acad Sci U S A 101:14782-14787.

Gautam M, Noakes PG, Mudd J, Nichol M, Chu GC, Sanes JR, Merlie JP (1995) Failure of postsynaptic specialization to develop at neuromuscular junctions of rapsyn-deficient mice. Nature 377:232-236.

Grady RM, Zhou H, Cunningham JM, Henry MD, Campbell KP, Sanes JR (2000) Maturation and maintenance of the neuromuscular synapse: genetic evidence for roles of the dystrophin-glycoprotein complex. Neuron 25:279-293.

Hain J, Onoue H, Mayrleitner M, Fleischer S, Schindler H (1995) Phosphorylation modulates the function of the calcium release channel of sarcoplasmic reticulum from cardiac muscle. J Biol Chem 270:2074-2081.

Hassink GC, Zhao B, Sompallae R, Altun M, Gastaldello S, Zinin NV, Masucci MG, Lindsten K (2009) The ER-resident ubiquitin-specific protease 19 participates in the UPR and rescues ERAD substrates. EMBO Rep 10:755-761.

Juo P, Kaplan JM (2004) The anaphase-promoting complex regulates the abundance of GLR-1 glutamate receptors in the ventral nerve cord of $C$. elegans. Curr Biol 14:2057-2062.

Kim N, Stiegler AL, Cameron TO, Hallock PT, Gomez AM, Huang JH, Hubbard SR, Dustin ML, Burden SJ (2008) Lrp4 is a receptor for Agrin and forms a complex with MuSK. Cell 135:334-342.

Lu Z, Je HS, Young P, Gross J, Lu B, Feng G (2007) Regulation of synaptic growth and maturation by a synapse-associated $\mathrm{E} 3$ ubiquitin ligase at the neuromuscular junction. J Cell Biol 177:1077-1089.

Mabb AM, Ehlers MD (2010) Ubiquitination in postsynaptic function and plasticity. Annu Rev Cell Dev Biol 26:179-210.

Martinez-Pena y Valenzuela I, Mouslim C, Akaaboune M (2010) Calcium/ calmodulin kinase II-dependent acetylcholine receptor cycling at the mammalian neuromuscular junction in vivo. J Neurosci 30:12455-12465.

Martinez-Pena y Valenzuela I, Mouslim C, Pires-Oliveira M, Adams ME, Froehner SC, Akaaboune M (2011) Nicotinic acetylcholine receptor stability at the NMJ deficient in $\alpha$-syntrophin in vivo. J Neurosci 31:15586-15596.

Nori A, Lin PJ, Cassetti A, Villa A, Bayer KU, Volpe P (2003) Targeting of alpha-kinase-anchoring protein (alpha KAP) to sarcoplasmic reticulum and nuclei of skeletal muscle. Biochem J 370:873-880.

Okada K, Inoue A, Okada M, Murata Y, Kakuta S, Jigami T, Kubo S, Shiraishi H, Eguchi K, Motomura M, Akiyama T, Iwakura Y, Higuchi O, Yamanashi Y (2006) The muscle protein Dok-7 is essential for neuromuscular synaptogenesis. Science 312:1802-1805.

Pawson T, Scott JD (1997) Signaling through scaffold, anchoring, and adaptor proteins. Science 278:2075-2080.

Radivojac P, Vacic V, Haynes C, Cocklin RR, Mohan A, Heyen JW, Goebl MG, Iakoucheva LM (2010) Identification, analysis, and prediction of protein ubiquitination sites. Proteins 78:365-380.

Rock KL, Gramm C, Rothstein L, Clark K, Stein R, Dick L, Hwang D, Goldberg AL (1994) Inhibitors of the proteasome block the degradation of most cell proteins and the generation of peptides presented on MHC class I molecules. Cell 78:761-771.

Sanes JR, Lichtman JW (2001) Induction, assembly, maturation and maintenance of a postsynaptic apparatus. Nat Rev Neurosci 2:791-805.

Schaefer AM, Hadwiger GD, Nonet ML (2000) rpm-1, a conserved neuronal gene that regulates targeting and synaptogenesis in C. elegans. Neuron $26: 345-356$ 
Singh P, Salih M, Tuana BS (2009) Alpha-kinase anchoring protein alphaKAP interacts with SERCA2A to spatially position $\mathrm{Ca}^{2+} /$ calmodulin-dependent protein kinase II and modulate phospholamban phosphorylation. J Biol Chem 284:28212-28221.

Sowa ME, Bennett EJ, Gygi SP, Harper JW (2009) Defining the human deubiquitinating enzyme interaction landscape. Cell 138:389-403.

Speese SD, Trotta N, Rodesch CK, Aravamudan B, Broadie K (2003) The ubiquitin proteasome system acutely regulates presynaptic protein turnover and synaptic efficacy. Curr Biol 13:899-910.

Wan HI, DiAntonio A, Fetter RD, Bergstrom K, Strauss R, Goodman CS (2000) Highwire regulates synaptic growth in Drosophila. Neuron 26:313-329.
Wang J, Best PM (1992) Inactivation of the sarcoplasmic reticulum calcium channel by protein kinase. Nature 359:739-741.

Weatherbee SD, Anderson KV, Niswander LA (2006) LDL-receptor-related protein 4 is crucial for formation of the neuromuscular junction. Development 133:4993-5000.

Wu H, Xiong WC, Mei L (2010) To build a synapse: signaling pathways in neuromuscular junction assembly. Development 137:1017-1033.

Xu A, Hawkins C, Narayanan N (1993) Phosphorylation and activation of the $\mathrm{Ca}^{2+}$-pumping ATPase of cardiac sarcoplasmic reticulum by $\mathrm{Ca}^{2+} /$ calmodulin-dependent protein kinase. J Biol Chem 268:8394-8397.

Zhang B, Luo S, Wang Q, Suzuki T, Xiong WC, Mei L (2008) LRP4 serves as a coreceptor of agrin. Neuron 60:285-297. 